\title{
EFECTO DEL NIVEL ALIMENTICIO SOBRE EL RENDIMIENTO Y CALIDAD DE FIBRA EN ALPACAS
}

\author{
The Effect of Two Levels of Feeding on the Yield and Quality of Alpaca \\ FiBRE
}

\author{
Francisco Franco F. ${ }^{1,2}$, Felipe San Martín H. ${ }^{3}$, Miguel Ara G. ${ }^{3}$, Juan Olazabal L. ${ }^{1}$ y \\ Fernando Carcelén $\mathrm{C}^{3}{ }^{3}$
}

\section{Resumen}

\begin{abstract}
El presente estudio se realizó en la Estación Experimental del Centro de Investigación IVITA-Maranganí con el objetivo de evaluar el efecto de alimentación de dos niveles contrastantes de energía sobre el rendimiento y calidad de fibra en alpacas. Se utilizó 12 alpacas Huacaya machos jóvenes. Se tuvo una fase pre-experimental para el acostumbramiento de los animales a una dieta balanceada y una fase experimental con cuatro periodos: los periodos I y III, de 28 días cada uno, se usaron para igualar condiciones corporales; en el periodo II los animales fueron sometidos a los tratamientos T1 y T2 ofreciéndoles 0.73 y 1.23 veces el requerimiento energético de mantenimiento por 84 días; y en el periodo IV los animales del T1 pasaron al T2 y viceversa por otros 84 días. En los periodos II y IV se tomaron muestras de fibra de un área de $100 \mathrm{~cm}^{2}$ sobre el costillar medio derecho, midiéndose el rendimiento, el diámetro (D), la longitud (L), la relación de L/ D, el volumen (V), y el aporte de L, D y (D)(L) en el V de la fibra. El T2 fue significativamente superior al T1 $(\mathrm{p}<0.05)$ en rendimiento de la fibra (5.22 vs. $\left.4.43 \mathrm{~g} / 100 \mathrm{~cm}^{2}\right), \mathrm{D}(25.75 \mathrm{vs} .23 .97$ $\mu), \mathrm{L}(319.6$ y $294.7 \mu / \mathrm{d})$ y V (162.8 vs. $132.95 \times 10^{-3} \mu / \mathrm{d}$, respectivamente), en tanto que la relación $\mathrm{L} / \mathrm{D}$ fue similar en ambos tratamientos. D, L y (D)(L) aportaron el 68.6, 27.2 y 4.2\% al $\mathrm{V}$ de la fibra, respectivamente. Se concluye que los niveles alimenticios en la alpaca influyen tanto en la producción como en el volumen de la fibra y que el aporte del D en el incremento de la fibra resultante es mayor que el aporte de L.
\end{abstract}

Palabras clave: alpaca, producción, fibra, nutrición

\section{Abstract}

The present study was carried out at the IVITA-Maranganí Experimental Station to evaluate the effect of two levels of feeding on the yield and quality of alpaca fibre. Twelve young male alpacas were used. A pre experimerntal phase was conducted for the adaptation of the animals to the balanced diet and an experimental phase with four periods was considered: Periods I and III of 28 days each were used to level body condition; in period

1 Estación Experimental del Centro de Investigación IVITA-Marangani, ${ }^{3}$ Laboratorio de Bioquímica, Nutrición y Alimentación Animal, Facultad de Medicina Veterinaria, Universidad Nacional Mayor de San Marcos, Lima

${ }^{2}$ E-mail:ffrancof@unmsm.edu.pe 
II the animals were submitted to treatment $\mathrm{T} 1$ and $\mathrm{T} 2$, receiving 0.73 and 1.23 times the maintenance requirement for 84 days; and in period IV the animals of T1 were shifted to $\mathrm{T} 2$ and vice versa for another 84 days. In periods II and IV, fiber samples were taken from a $100 \mathrm{~cm}^{2}$ area on the right mid rib area, and was measured the yield, diameter (D), length (L), volume (V), L/D ratio and the contribution of L, D and (D)(L) on fibre volume. T2 was statistically superior to T1 $(\mathrm{p}<0.05)$ in fibre yield $\left(5.22 \mathrm{vs.} 4.43 \mathrm{~g} / 100 \mathrm{~cm}^{2}\right), \mathrm{D}(25.75 \mathrm{vs}$. $23.97 \mu), \mathrm{L}(319.6$ y $294.7 \mu / \mathrm{d})$ and V (162.8 vs. $132.95 \times 10^{-3} \mu / \mathrm{d}$ respectively) while the L/ $\mathrm{D}$ ratio was similar in both treatments. D, L and (D)(L) contributed with 68.6, 27.2 and $4.2 \%$ to fibre volume, respectively. It is concluded that the feeding levels in the alpaca influenced both the yield and volume of fibre, and that the contribution of $\mathrm{D}$ in the fibre volume resulted higher than the L contribution.

Key words: alpaca, production, fiber, nutrition

\section{INTRODUCCIÓN}

La producción y la calidad de la fibra en alpacas se afectan por el medio ambiente (estación, fotoperiodo, temperatura, altitud), la genética (individuo, raza, edad) y el estado fisiológico del animal (lactancia, preñez). En el país, uno de los factores de mayor importancia que afecta el rendimiento de la fibra es el estado de subnutrición en ciertos periodos del año. Así, las praderas, base de la alimentación de los camélidos sudamericanos (CSA), presentan deficiencia de energía al inicio del periodo de lluvias, deficiencia de proteína al inicio del periodo seco y deficiencia de energía y proteína en la época seca propiamente dicha (San Martín, 1996). Estos periodos de subnutrición son seguidos por periodos de relativa abundancia de alimento, los cuales ejercen un efecto marcado sobre la producción y calidad de la fibra.

Estudios acerca del efecto de la nutrición sobre el crecimiento y calidad de la fibra en alpacas son escasos. Agramonte (1988) observó una mayor producción y mayor diámetro de fibra en animales criados en pasturas cultivadas que en praderas. Russel y Redden (1997) sometieron a un grupo de alpacas a un régimen nutricional de submantenimiento $(0.7 \mathrm{M})$ seguido por otro de sobremantenimiento $(2 \mathrm{M})$, encontrando que la alpaca es altamente sensible a la manipulación nutricional y que su efecto sobre la producción de fibra se ejerce más a través de cambios en el largo de la fibra que en el diámetro, en contraste con lo hallado en ovinos (Reis y Sahlu, 1994). Las contradicciones de los resultados en los pocos estudios realizados para medir el efecto de la nutrición sobre la fibra de las alpacas llevaron a plantear este trabajo, cuyo propósito es dilucidar el efecto de niveles nutricionales contrastantes sobre el rendimiento y calidad de la fibra.

\section{Materiales y Métodos}

\section{Lugar del Estudio}

El trabajo de campo se llevó a cabo en el fundo San Marcos de la Estación Experimental del Centro de Investigación IVITAMaranganí, Facultad de Medicina Veterinaria, Universidad Nacional Mayor de San Marcos. El fundo está ubicado en el departamento del Cusco, provincia de Canchis. La zona tiene temperaturas máximas entre 13 a $15^{\circ} \mathrm{C}$ y mínimas entre -7 a $2.5^{\circ} \mathrm{C}$, y con un promedio de precipitación pluvial anual de $952 \mathrm{~mm}$ y con variaciones de 90 a $200 \mathrm{~mm} / \mathrm{mes}$. El estudio se desarrolló entre junio de 2003 a enero de 2004. 


\section{Animales Experimentales}

Se emplearon 12 alpacas Huacaya machos, de 3 a 4 años de edad, color blanco, peso promedio de $60 \mathrm{~kg}$, seleccionadas al azar de una majada alimentada con pastos nativos donde se presentaba dominancia de la asociación de Festuca rigida y Muhlembergia fastigiata (Feri-Mufa). Se contó con una fase pre-experimental de 14 días, en la que se ofreció a los animales, para su acostumbramiento, el alimento base experimental (ABE), con $2,589 \mathrm{kcal}$ de energía metabolizable/kg MS, y $12.5,17.1,5.5,8.2$ y $56.7 \%$ de proteína, fibra, extracto etéreo, cenizas y extracto libre de nitrógeno, respectivamente. El alimento y el agua fueron proporcionados ad libitum en comederos y bebederos en cubículos individuales.

La fase experimental tuvo cuatro periodos:

Periodo I. 28 días. Los animales recibieron el ABE en cantidades suficientes para cubrir sus requerimientos de mantenimiento $(1 \mathrm{M}) \mathrm{y}$ poder igualar sus condiciones corporales. El requerimiento energético y proteico de nutrientes calculado fue de $114 \mathrm{kcal}$ de EM/ kg PM [25\% más del estimado por Carmean et al. (1992)] y $118 \mathrm{~g}$ de proteína [25\% más del estimado por San Martín (1991)].

Periodo II. 84 días. Al inicio del periodo, todos los animales fueron pesados y rasurados a la altura del costillar medio en un área de $100 \mathrm{~cm}^{2}$ (área de estudio de la fibra). A la mitad de los animales (T1) se les ofreció el equivalente a 0.73 veces el requerimiento de mantenimiento $(0.7 \mathrm{M})$ y a la otra mitad (T2) el equivalente a 1.23 veces dicho requerimiento $(1.2 \mathrm{M})$, a fin de tener regímenes nutricionales contrastantes (baja y alta nutrición). Se tomó muestras de la fibra y los animales se pesaron cada 28 días (subperiodos) hasta completar tres muestreos.

Periodo III. 28 días. Todos los animales recibieron el ABE en cantidades suficientes para cubrir sus requerimientos de mantenimiento (1 M). Al fin del periodo, los animales fueron rasurados y pesados.
Periodo IV. 84 días. Los animales que recibieron el alimento del $\mathrm{T} 1$ en el periodo II pasaron al T2 y viceversa. Se tomó muestras de la fibra y los animales se pesaron cada 28 días (subperiodos) hasta completar tres muestreos.

\section{Observaciones Realizadas}

Peso vivo. El pesaje de los animales se hizo en ayunas a la misma hora (07:00) usando una balanza tipo reloj, con capacidad de 150 kg y una aproximación de $500 \mathrm{~g}$, acondicionada a un trípode.

Producción de fibra. Se evaluó un área de $100 \mathrm{~cm}^{2}$ sobre la parte media del costillar derecho. El margen superior se ubicó a 20 $\mathrm{cm}$ de la columna vertebral y el margen derecho sobre la última costilla, siguiendo la técnica descrita por Braga (1987) y Villarroel (1963). Las muestras de fibra sucia fueron pesadas para obtener el peso de la fibra sucia. La fibra limpia fue obtenida por el pasaje en soluciones tibias $\left(40^{\circ} \mathrm{C}\right)$ de detergente, bicarbonato de soda y agua. Las muestras fueron desecadas en estufa a $100{ }^{\circ} \mathrm{C}$ por dos horas y luego acondicionadas en campanas desecadoras con silica gel, por espacio de 15 min dentro de un ambiente controlado a $23{ }^{\circ} \mathrm{C}$ y $67 \%$ de humedad relativa. Luego se registró el peso de la fibra limpia, siguiendo la técnica descrita por García et al. (2006).

Longitud de fibra (L). La L de las muestras de fibra limpia fueron obtenidas con una regla milimetrada y expresadas en micras (mm).

Diámetro de fibra (D). Las muestras de fibra limpia fueron cortadas con un micrómetro y montadas en láminas portaobjetos con bálsamo de Canadá. El D se midió después 48 h utilizando un lanámetro y el proceso estándar IWTO 8-89-E (IWTO, 1989).

Otras observaciones. La relación L/D, el volumen de fibra (V), y el aporte de L, D y (D)(L) al incremento de V se basaron en los cálculos descritos por Reis y Sahlu (1994). 


\section{Análisis de Datos}

Se empleó un diseño de sobrecambio simple con dos periodos (Periodo II y Periodo IV) y dos tratamientos (T1 y T2) con seis alpacas cada uno. El análisis de datos se realizó con ayuda del paquete estadístico SAS (1990). Las diferencias entre tratamientos fueron evaluadas mediante el cálculo del ANOVA correspondiente y las pruebas de $\mathrm{F}$ respectivas. Adicionalmente, se evaluaron las diferencias entre tratamientos dentro de cada subperiodos de 28 días.

\section{Resultados}

El efecto de los tratamientos sobre el peso vivo se muestra en el Cuadro 1 . Los animales del T1 $(0.7 \mathrm{M})$ perdieron $40.1 \mathrm{~g} / \mathrm{d}$ mientras que los animales del T2 $(1.2 \mathrm{M})$ ganaron $51.7 \mathrm{~g} / \mathrm{d}(\mathrm{p}<0.05)$.

El rendimiento de fibra limpia fue $17.8 \%$ superior en el T2 con relación al T1 ( $\mathrm{p}<0.05$, Cuadro 1). Cuando se dividen los periodos II y IV en subperiodos de 28 días (Cuadro 2), se puede observar que la producción de fibra limpia empieza a diferenciarse en el subperiodo 2 en ambos tratamientos $(\mathrm{p}<0.05)$, siendo superior el T2 con respecto al T1 en 29.3 y $25.2 \%$ en los subperiodos 2 y 3 , respectivamente. Por otro lado, en el T2, la producción se incrementa al pasar del subperiodo 1 al subperiodo 2 (14\%) y de este último al subperiodo 3 (32\%), mientras que en el T1 se observa una disminución de la producción entre el subperiodo 1 y 2 (12\%), recuperándose al pasar al subperiodo 3 .

E1 T2 mostró una mayor longitud de fibra limpia que el T1 $(8.5 \%, \mathrm{p}<0.05$, Cuadro 1). Al realizar el análisis por subperiodos, se encontró diferencia significativa entre tratamientos en los subperiodos 2 y $3(\mathrm{p}<0.05$, Cuadro 2). Así mismo, no se observa diferencias significativas entre subperiodos dentro del T2, mientras que en el T1, en forma similar a la producción de fibra, se observa una reducción de la longitud (6.8\%) entre el subperiodo 1 y 2 , recuperándose al pasar al subperiodo 3.

El T2 mostró un mayor diámetro de fibra limpia que el T1 (7.4\%, $\mathrm{p}<0.05$, Cuadro 1). Al analizar por superiodos, se observa que el D se mantiene constante en T1; mientras que en T2 aumenta en 13.8 y $19.4 \%$ al comparar los subperiodos 2 y 3 con el 1 , respectivamente (Cuadro 2).

La relación L/D se mantuvo constante debido a que el efecto del tratamiento produjo similares variaciones en ambas variables (Cuadros 1 y 2).

Se obtuvo el $22.4 \%$ de incremento de volumen de fibra en el $\mathrm{T} 2$ con respecto al $\mathrm{T} 1$ $(\mathrm{p}<0.05$, Cuadro 1). Al analizar por subperiodos se observa que en el subperiodo 1 el volumen es similar, pero en los subperiodos 2 y 3 aumenta en un 40\% (Cuadro 3). El aporte del D fue mayor (68.6\%) que el aporte de la L (27.2\%) y del (D)(L) (4.2\%) en el volumen de la fibra. Asimismo, se observa que el aporte de la $\mathrm{L}$ y $\mathrm{D}$, al pasar del subperiodo 2 al 3 disminuye y aumenta en 25.8 y 28.9 unidades, respectivamente, mientras que el aporte del (D)(L) disminuye en 3.1 unidades.

\section{Discusión}

Las ganancias de peso halladas en el presente trabajo (Cuadro 1) fueron inferiores a las obtenidas por Russel y Redden (1997) quienes sometiendo a los animales a consumos similares a los del presente estudio obtuvieron ganancias de peso de $-69.1 \mathrm{y}$ $70.3 \mathrm{~g} / \mathrm{d}$, respectivamente. Estas diferencias podrían explicarse por la edad de los animales, pues en el presente estudio se usaron alpacas jóvenes de 3 a 5 años con un peso promedio de $60 \mathrm{~kg}$ mientras que en el otro estudio eran animales adultos con un peso promedio de $74 \mathrm{~kg}$. 
Cuadro 1. Rendimiento productivo de alpacas sometidas a dos niveles contrastantes de alimentación

\begin{tabular}{lcc}
\hline \multirow{2}{*}{ Variables } & \multicolumn{2}{c}{ Tratamientos } \\
\cline { 2 - 3 } & $\mathrm{T} 1^{1}$ & $\mathrm{~T} 2^{2}$ \\
\hline Ganancia de peso vivo, $(\mathrm{g} / \mathrm{d})$ & $-40.1^{\mathrm{a}}$ & $51.7^{\mathrm{b}}$ \\
Fibra limpia 0-12 semanas, $\left(\mathrm{g} / 100 \mathrm{~cm}^{2}\right)$ & $4.43^{\mathrm{a}}$ & $5.22^{\mathrm{b}}$ \\
Longitud de fibra $(\mathrm{L}),(\mu \mathrm{m} / \mathrm{d})$ & $294.7^{\mathrm{a}}$ & $319.6^{\mathrm{b}}$ \\
Diámetro de la fibra $(\mathrm{D}),(\mu \mathrm{m})$ & $23.97^{\mathrm{a}}$ & $25.75^{\mathrm{b}}$ \\
Relación L / D & $12.52^{\mathrm{a}}$ & $12.63^{\mathrm{a}}$ \\
Volumen, $\left(\mu \mathrm{m}^{3} / \mathrm{d}\right)$ & $132.95^{\mathrm{a}}$ & $162.79^{\mathrm{b}}$ \\
\hline
\end{tabular}

${ }^{1} \mathrm{~T} 1: 0.73$ veces el requerimiento de mantenimiento

${ }^{2}$ T2: 1.23 veces el requerimiento de mantenimiento

${ }^{a, b}$ Superíndices diferentes dentro de filas indican diferencia estadística $(p<0.05)$

La cantidad producida de fibra limpia coincide con resultados encontrados en la literatura en alpacas (Newman y Paterson, 1994; Russel y Redden, 1997; Bustinza, 2001; García et al., 2006) como en ovinos (Downes y Sharry, 1971; Henderson, 1980; Hynd y Masters, 2002). Al analizar los resultados por subperiodos, es notoria la disminución de producción de fibra entre los subperiodos 1 y 2 del T1; sin embargo, se logra una ligera recuperación en el subperiodo 3 , tal vez debido a que los animales se adaptan a las condiciones de restricción nutricional y movilizan nutrientes de otros tejidos corporales a la lana (Doyle y Egan, 1983). Por otro lado, los incrementos entre subperiodos en el T2 coinciden con lo reportado en cabras (Shahjalal et al., 1992).

El hecho de no haber una recuperación en la producción de fibra al final del primer mes (subperiodo 1) de la sobrealimentación (T2) se debe a que los cambios de la masa folicular son inicialmente más lentos por la competencia de nutrientes con otros tejidos que tienen mayores demandas de nutrientes (músculo e intestinos) (Liu et al., 1998;
Adams et al., 2000). Esto supondría que la recuperación de la producción de la fibra luego de un periodo de restricción alimenticia solo se lograría después del primer mes de una mejora en la calidad de la alimentación. Por otro lado, la recuperación de la producción de fibra no contempla el fenómeno del crecimiento compensatorio o aumento de la tasa de crecimiento de la lana como ocurre con la ganancia de peso (Butler-Hogg, 1984).

Los resultados de L y D de la fibra limpia muestran una tendencia similar con los de Russel y Redden (1997), aunque en este caso los resultados fueron inferiores. Los resultados por subperiodos de la $\mathrm{L}$ de la fibra mostraron una tendencia similar a la descrita para la producción de fibra limpia, ya que la L de fibra está relacionada, además del tamaño de bulbo folicular, a la longitud de las células corticales y a la proporción de células que ingresan a la propia fibra (Hynd, 1994; Hynd y Masters, 2002). Por otro lado, las diferencias en D de fibra con los resultados de Russel y Redden (1997) pueden deberse a la edad, pues se conoce que el D se incrementa con la edad (Calderón y Pumayala, 1981; 
Cuadro 2. Producción, longitud (L), diámetro (D) y relación $\mathrm{L} / \mathrm{D}$ de fibra limpia en alpacas sometidas a dos niveles contrastantes de alimentación empleando un diseño de sobrecambio simple con dos periodos

\begin{tabular}{|c|c|c|c|c|}
\hline \multirow{2}{*}{ Variable } & \multicolumn{2}{|c|}{ Subperiodo } & \multicolumn{2}{|c|}{ Tratamientos } \\
\hline & $\mathrm{N}^{\mathrm{o}}$ & Días & $\mathrm{T} 1^{1}$ & $\mathrm{~T} 2^{2}$ \\
\hline \multirow{4}{*}{$\begin{array}{l}\text { Producción de fibra } \\
\left(\mathrm{g} / 100 \mathrm{~cm}^{2}\right)\end{array}$} & 1 & $0-28$ & $1.51^{\mathrm{a}}$ & $1.51^{\mathrm{a}}$ \\
\hline & 2 & $29-56$ & $1.33^{\mathrm{a}}$ & $1.72^{\mathrm{b}}$ \\
\hline & 3 & $57-84$ & $1.59^{\mathrm{a}}$ & $1.99^{\mathrm{b}}$ \\
\hline & Promedio & $0-84$ & $4.43^{\mathrm{a}}$ & $5.22^{\mathrm{b}}$ \\
\hline \multirow{4}{*}{$\begin{array}{l}\text { Longitud de fibra } \\
(\mu / d)\end{array}$} & 1 & $0-28$ & $299.2^{\mathrm{a}}$ & $311.4^{\mathrm{a}}$ \\
\hline & 2 & $29-56$ & $278.9^{\mathrm{a}}$ & $321.9^{\mathrm{b}}$ \\
\hline & 3 & $57-84$ & $305.8^{\mathrm{a}}$ & $324.2^{\mathrm{b}}$ \\
\hline & Promedio & $0-84$ & $294.6^{\mathrm{a}}$ & $319.6^{\mathrm{b}}$ \\
\hline \multirow{4}{*}{$\begin{array}{l}\text { Diámetro de fibra } \\
(\mu)\end{array}$} & 1 & $0-28$ & $23.89^{\mathrm{a}}$ & $23.18^{\mathrm{a}}$ \\
\hline & 2 & $29-56$ & $24.09^{\mathrm{a}}$ & $26.37^{b}$ \\
\hline & 3 & $57-84$ & $23.94^{\mathrm{a}}$ & $27.70^{\mathrm{b}}$ \\
\hline & Promedio & $0-84$ & $23.97^{\mathrm{a}}$ & $25.75^{\mathrm{b}}$ \\
\hline \multirow{4}{*}{ Relación L/D } & 1 & $0-28$ & $12.81^{\mathrm{a}}$ & $13.54^{\mathrm{a}}$ \\
\hline & 2 & $29-56$ & $11.80^{\mathrm{a}}$ & $12.45^{\mathrm{a}}$ \\
\hline & 3 & $57-84$ & $12.96^{\mathrm{a}}$ & $12.03^{\mathrm{a}}$ \\
\hline & Promedio & $0-84$ & $12.52^{\mathrm{a}}$ & $12.67^{\mathrm{a}}$ \\
\hline
\end{tabular}

${ }^{1} \mathrm{~T} 1: 0.73$ veces el requerimiento de mantenimiento

${ }^{2}$ T2: 1.23 veces el requerimiento de mantenimiento

${ }^{a, b}$ Superíndices diferentes dentro de filas indican diferencia estadística $(p<0.05)$

Bustinza, 2001; Davis, 2001). La relación L/ D en el presente trabajo (Cuadro 2) se mantuvo constante, coincidiendo con otros reportes en ovinos (Downes y Sharry, 1971; Reis y Sahlu, 1994), aunque también hay resultados divergentes con otros trabajos en cabras Angora (Sahlu y Fernández, 1992; Sahlu et al., 1992).
El incremento de volumen de fibra por efecto de la mejora en la alimentación fue similar al reportado por Russel y Redden (1997); sin embargo, difieren en los niveles de contribución de la L, el D y (D)(L), toda vez que ellos encuentran que la contribución de L, D y $\mathrm{D}(\mathrm{L})$ fue de 79,18 y $3 \%$, respectivamente, a diferencia de este estudio donde 
Cuadro 3. Contribución de la longitud (L), diámetro (D) y (D)(L) al incremento de volumen de la fibra limpia de alpaca por efecto de dos niveles contrastantes de alimentación empleando un diseño de sobrecambio simple con dos periodos

\begin{tabular}{|c|c|c|c|c|c|c|c|}
\hline \multicolumn{2}{|c|}{ Sub periodo } & \multicolumn{2}{|c|}{ Tratamientos } & \multirow{2}{*}{$\begin{array}{l}\text { Incremento } \\
\text { de volumen }\end{array}$} & \multicolumn{3}{|c|}{ Incremento debido a: } \\
\hline & & $\mathrm{T} 1^{1}$ & $\mathrm{~T} 2^{2}$ & & $\mathrm{~L}$ & $\mathrm{D}$ & $(\mathrm{D})(\mathrm{L})$ \\
\hline 1 & \multirow{4}{*}{$\begin{array}{l}\text { Volumen } \\
\left(\mu^{3} / \text { día }\right)\end{array}$} & $134.1^{\mathrm{a}}$ & $131.4^{\mathrm{a}}$ & -2.7 & & & \\
\hline 2 & & $127.1^{\mathrm{a}}$ & $175.8^{\mathrm{a}}$ & 48.7 & 19.6 & 25.2 & 3.9 \\
\hline 3 & & $137.7^{\mathrm{a}}$ & $195.5^{\mathrm{a}}$ & 57.9 & 8.4 & 46.6 & 2.8 \\
\hline Promedio & & $133.0^{\mathrm{a}}$ & $162.8^{\mathrm{b}}$ & 29.8 & 8.1 & 20.5 & 1.2 \\
\hline 1 & \multirow{4}{*}{$\begin{array}{l}\text { Incremento } \\
\text { proporcional } \\
(\%)\end{array}$} & & & -2.0 & & & \\
\hline 2 & & & & 38.3 & 40.3 & 51.7 & 8.0 \\
\hline 3 & & & & 42.3 & 14.5 & 80.6 & 4.9 \\
\hline Promedio & & & & 22.4 & 27.2 & 68.6 & 4.2 \\
\hline
\end{tabular}

la mayor contribución estuvo dada por el D $(68.7 \%)$ seguida por la L $(27.2 \%)$ y la (D)(L) (4.2\%). No obstante estas diferencias, los resultados concuerdan con estudios llevados a cabo en ovinos Merino donde la contribución del $\mathrm{D}$ y de la $\mathrm{L}$ en el volumen de la fibra fue del $54-56 \%$ y de $26-31 \%$, respectivamente (Reis, 1992; Hynd y Masters, 2002).

\section{Conclusiones}

- El nivel alimenticio en la alpaca influye tanto en la producción como en el volumen de la fibra.

- El aporte del diámetro en el incremento del volumen de la fibra resultante es mayor que el aporte de la longitud, tal como sucede en ovinos y cabras.
- La respuesta negativa o positiva del nivel alimenticio en la producción de fibra se observa a partir del segundo mes cuando la alpaca es alimentada con un nivel de 0.7 o 1.23 veces el necesario para cubrir sus requerimientos de mantenimiento.

\section{Literatura Citada}

1. Adams N, Liu S, Masters D. 2000. Regulation of protein synthesis of wool growth. In: Cronjé PB. Ruminant physiology. New York: CABI Publishing. p 255-276.

2. Agramonte M. 1988. Incremento del peso corporal de crías y ritmo de crecimiento de la fibra de alpacas en dos sistemas de producción. Tesis de Ingeniero Zootecnista. Cusco: Univ. Nacional San Antonio Abad del Cusco. 72 p. 
3. Braga $W .1987$. El efecto de la altitud en la producción de fibra de la alpaca (Lama pacos). Tesis de Médico Veterinario. Lima: Univ. Nacional Mayor de San Marcos. 93 p.

4. Bustinza V. 2001. La alpaca, conocimiento del gran potencial andino. Puno: Univ. Nacional del Altiplano. $343 \mathrm{p}$.

5. Butler-Hogg $B W$. 1984. Growth patterns in sheep: Wool growth during weight loss and subsequent compensatory growth. J Agric Sci, Cambridge 102: 105-109.

6. Calderón A, Pumayala A. 1981. Efectos de la edad sobre la longitud de la mecha, peso de vellón y peso vivo en alpacas Huacaya. En: IV Reunión APPA. Ayacucho: Asociación Peruana de Producción Animal.

7. Carmean B, Johnson K, Johnson D. 1992. Maintenance energy requirement of llamas. Am J Vet Res 53: 1696-1698.

8. Davis GH. 2001. Some factors affecting fiber production in alpacas. Christchurch New Zealand: Proc AAA (NZ) Conference. p 1-20.

9. Downes AM, Sharry LF. 1971. Measurement of wool growth and its response to nutritional changes. Aust $\mathrm{J}$ Biol Sci 24: 117-130.

10. Doyle P, Egan J. 1983. The utilization of nitrogen and sulfur by weaner and mature sheep. Aust J Agric Res 34: 433439.

11. García W, Olazábal J, Franco F, Salazar A. 2006. Variación de la finura y crecimiento de la fibra en alpacas Suris en función a la edad y época. En: II Simposio Internacional de Investigaciones sobre CSA. Arequipa, Perú. p 177-183.

12. Henderson $\boldsymbol{A E}$. 1980. Nutrition and quantitative wool growth. Lincoln, New Zealand: Lincoln College Univ. 268 p.

13. Hynd PI. 1994. Follicular determinants of the length / diameter ratio at two levels of nutrition. Aust J Agric Res 45: 11371147.
14. Hynd PI, Masters DG. 2002. Nutrition and wool growth. In: Freer M, Dove H. (eds). Sheep nutrition. Australia: CSIRO. p 165-185.

15. [IWTO] International Wool Textile Organisation. 1989. Specifications IWTO-8-89(E). Ilkley, UK: International Wool Secretariat. $32 \mathrm{p}$.

16. Liu S, Mata G, O'Donoghue M, Masters D. 1998. The influence of live weight, live weight change and diet on protein synthesis in the skin and skeletical muscle in young merino sheep. Brit J Nut 79: 267-274.

17. Newman SA, Paterson D. 1994. Effect of level of nutrition and season on fibre growth in alpacas. Proc New Zeal Soc Anim Prod 54: 147-150.

18. Reis PJ. 1992. Variations in the strength of wool fibres - a review. Aust J Agric Res 43: 1337-1351.

19. Reis PJ, Sahlu T. 1994. The nutritional control of the growth and properties of mohair and wool fibers: a comparative review. J Anim Sci 72: 1899-1907.

20. Russel AJF, Redden HL. 1997. The effect of nutrition on fibre growth in the alpaca. J Anim Sci 64: 509-512.

21. Sahlu T, Fernandez JM. 1992. Effect of intraperitoneal administration of lysine and methionine on mohair yield and quality in Angora goats. J Anim Sci 70: 3188-3193.

22. Sahlu, T, Fernandez JM, Lu CD, Manning L. 1992. Dietary protein level and ruminal degradability for mohair production in Angora goats. J Anim Sci 70: 1526-1533.

23. San Martín F. 1991. Nutrición y alimentación en alpacas y llamas. En: Novoa C (ed). Producción de rumiantes menores. Lima: Programa de apoyo en la investigación - Rumiantes menores. p 72-99.

24. San Martín F. 1996. Nutrición en alpacas y llamas. Fondo Contravalor Perú-Suiza, CISA/IVITA, Fac. Med. Vet., Univ. Nac. Mayor San Marcos. Pub. Cient. IVITA N ${ }^{\circ}$ 27: 28 p. 
25. [SAS] Statistical Analysis System. 1990. SAS/STAT User's Guide. Version 6. $4^{\text {th }}$. SAS Insitute.

26. Shahjalal M, Galbrait H, Topps J. 1992. The effect of changes in dietary protein and energy on growth, body com- position and mohair fibre characteristics of British Angora goats. J Anim Prod 54: 405-412.

27. Villarroel J. 1963. Un estudio de la fibra de alpaca. Anales Cient. Univ. Nac. Agraria La Molina, Lima 3: 247-273. 